\title{
Prostaglandin E Synthase Measurement
}

National Cancer Institute

\section{Source}

National Cancer Institute. Prostaglandin E Synthase Measurement. NCI Thesaurus. Code C103433.

The determination of the amount of the prostaglandin E synthase in a sample. 